\title{
FEM Analysis of Double Legs Bracket to Reduce the Local Stress Concentration for Automobile Applications
}

\author{
Dong Hee Kanga , Hyun Wook Kanga,, ${ }^{\mathrm{a}}$, Hyun-Taeck Limb, Hee-Sung Yoon, \\ Byung-Wook Kwon ${ }^{c}$, Sang-Duck Kim ${ }^{\mathrm{c}}$ \\ aDepartment of Mechanical Engineering, Chonnam National University, \\ 77 Youngbong-ro, Buk-Gu, Gwangju 61186, Republic of Korea \\ bReliability \& Safety R\&D Center, Korea Automotive Technology Institute, \\ 303 Pungse-ro, Pungse-myeon, Dongnam-gu, Chenan-si, Chungnam 31214, Republic of Korea \\ 'Yura Tech, 38 Anmosan-gil, Jeonui-myeon, Sejong-si, Republic of Korea \\ *Corresponding Author: kanghw@chonnam.ac.kr
}

\begin{abstract}
Generally, vehicle sensor on bracket is installed in engine room to receive sensing signals directly. The brackets in the engine room are exposed to extreme environmental conditions such as high temperature and irregular vibrations. For ease of production, traditional bracket structure has cantilever frame with one leg and two fixed points that is vulnerable to local stress concentration. High local stress in the bracket structure results in malfunctions and shorten life span. From these points, we suggest double legs and two fixed points bracket model with various hinge structures for local stress decreasing. To analysis the stress distribution and working conditions, vibration durability tests are performed for traditional and modified bracket models via finite element method. Impact hammer and grid tests are performed to validate the FEM analysis. Maximum stress conditions are evaluated with resonance frequency vibration analyses. As a result, the maximum stress on double legs bracket with increased thickness and radius of curvature model is reduced to $51.0 \%$ compared than traditional single leg bracket model.
\end{abstract}

Keywords: finite element method, resonance frequency, vibration, durability test, local stress concentration

\section{Introduction}

These days, vehicle development trend is changed to increasing the smart functions for intelligent vehicles. Compared with traditional vehicles, the intelligent vehicle needs many electric and electronic components for controlling and sensing the vehicle working conditions. ${ }^{(1-4)}$ Most of the electronic components are sensors that are installed in the engine room with bracket structures. ${ }^{(5,6)}$ The brackets are exposed to extreme environmental conditions such as high temperature and irregular vibrations. Due to the convenience of production, traditional bracket structure has single leg (SL) and two holes for fixing. According to these structural features, the traditional bracket is in danger to fracture due to high local stress at center hinge point between two fixed holes. ${ }^{(7,8)}$ Considering the importance of electronic components in intelligent vehicles, this potential for defects can lead to serious failure and damage to property and human life. In this research, to decrease the local stress intensity and ensure the operation stability and life span, modified bracket structures with double legs (DL) are investigated with various hinge structures such as increasing fillet radius, cogging, and flange beads on bracket legs. The vibration durability test is performed for traditional and modified brackets using finite element method (FEM) analysis. To simulate the extreme working conditions, vibrations are applied at the resonance frequencies with several accelerations for each bracket structures. Resonance frequencies are examined via experimental impact hammer test and numerical approach and validated each other. The local stress intensities and points are investigated through the FEM analysis and presented as important design parameters for bracket design.

\section{Numerical Analysis}

\subsection{Vibration conditions for stress analysis}

The stress analysis of bracket is based on durability test (C) 2018 The Institute of Industrial Applications Engineers, Japan. 


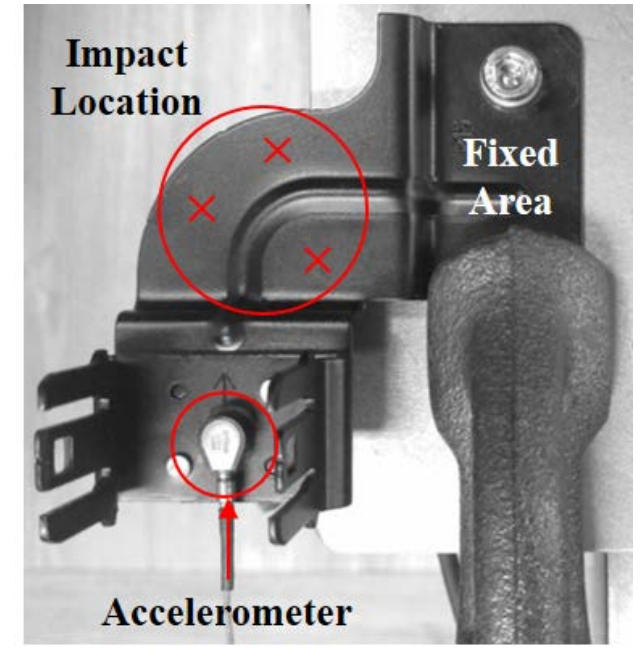

Figure 1. Schematic of single leg bracket for impact hammer test to search resonance frequencies.

Table 1. Identification of resonance frequencies in impact hammer and grid test results under $1 \mathrm{kHz}$.

\begin{tabular}{ccccccc}
\hline & \multicolumn{2}{l}{ Impact hammer test (Node) } & \multicolumn{3}{c}{ Grid test (Mesh No.) } \\
\cline { 2 - 7 } Mode & Node1 & Node2 & Node3 & $12.8 \mathrm{k}$ & $115.8 \mathrm{k}$ & $167.3 \mathrm{k}$ \\
\hline 1 & 149.9 & 150.3 & 150.3 & 145.17 & 142.39 & 142.32 \\
2 & 336.6 & 336.6 & 337.0 & 355.12 & 345.81 & 345.58 \\
3 & 671.6 & 672.4 & 672.8 & 757.7 & 734.46 & 734.05 \\
4 & 928.5 & 931.7 & 930.5 & 1022 & 989.36 & 988.66 \\
\hline
\end{tabular}

that is one of the environmental test standards for automobile electronics in the vibration condition. The durability test is performed to confirm the abnormality of model under the resonance frequency vibration. The resonance frequency searching test is performed on electronic parts for vehicles between 10 to $1000 \mathrm{~Hz}$. The resonance point obtained from the searching test result is applied for stress analysis. The specific shape in vibration at resonance point is called mode shape.

The vibration accelerations are applied $3 \mathrm{G}$ (G, acceleration in gravitational field, $\left.1 \mathrm{G}=9.81 \mathrm{~m} / \mathrm{s}^{2}\right), 1 \mathrm{G}$, and $0.5 \mathrm{G}$ for the resonance frequency range of 10 to $50 \mathrm{~Hz}, 50$ to $100 \mathrm{~Hz}$, and 100 to $1000 \mathrm{~Hz}$ respectively. If a resonance frequency is out of 10 to $1000 \mathrm{~Hz}$, the vibration acceleration is applied $3 \mathrm{G}$ with $33 \mathrm{~Hz}$. During the numerical analysis, the vibration acceleration is applied to 4 cycles of sine wave as the base excitation with $\mathrm{x}, \mathrm{y}$ and $\mathrm{z}$ directions. Also, the weight of electronic components and bracket is applied as body force with gravitational acceleration.

\subsection{Impact hammer and grid test before FEM stress analysis}

The impact hammer test is performed on bracket surface at three nodes using plastic tip hammer as shown in fig. 1 to obtain the resonance frequency of bracket. The vibration characteristics of bracket are obtained through the accelerometer under $2 \mathrm{kHz}$. For a numerical analysis, a grid test of bracket is performed to find exact resonance frequency. As shown in table 1, more than $100 \mathrm{k}$ number of meshes are used for numerical analysis to minimize the numerical errors.

\subsection{Mechanical properties and designs of bracket}

Most of brackets are made of cold-rolled steel sheet which is commonly used as vehicle chassis materials. During the numerical simulation, mechanical property of a coldrolled steel sheet is $200 \mathrm{MPa}$ of yield stress. However, bracket design is also necessary to consider of safety factor due to the extreme vibration environment. Safety factor (Safety factor, $\mathrm{S}$ = yield stress/ working stress) is a margin of security against several risks such as loads of static, cyclic and impact. From the Unwin's safety factor, safety factor of the cold-rolled steel in cyclic load condition is 8 that the maximum stress is limited to $25 \mathrm{MPa}$ in bracket. That is, the ratio of limited maximum stress / yield stress is $12.5 \%{ }^{(9)}$.

In fig. 2 shows stress distribution and stress variation at hinge point in single leg bracket that model is used for validation of stress analysis in FEM. Most of the distributed stresses are calculated under $1 \mathrm{MPa}$. However, 33.38 MPa of maximum stress is obtained at the center hinge point between two fixed holes, with $y$-direction acceleration in first point of resonance frequency (mode 1) condition. The ratio of maximum stress to yield stress is $16.7 \%$ that is too high to apply in vehicle. This potential for defects can lead to failure in cyclic vibration. A double legs bracket with additional hinge structures is considered to decrease the local stress intensity and ensure the operation stability and life span such as increased fillet radius, cogging, and flange beads on bracket legs. The bracket in fig. 3 shows the modified designs of double legs bracket with additional reinforced structures on bracket legs. The original model of DL-M1 has $2 \mathrm{~mm}$ of thickness and $3 \mathrm{~mm}$ of filet radius. To find the effects of additional process on structure legs, DL-M2 has cogging structure based on DL-M1 model. To find the effects 

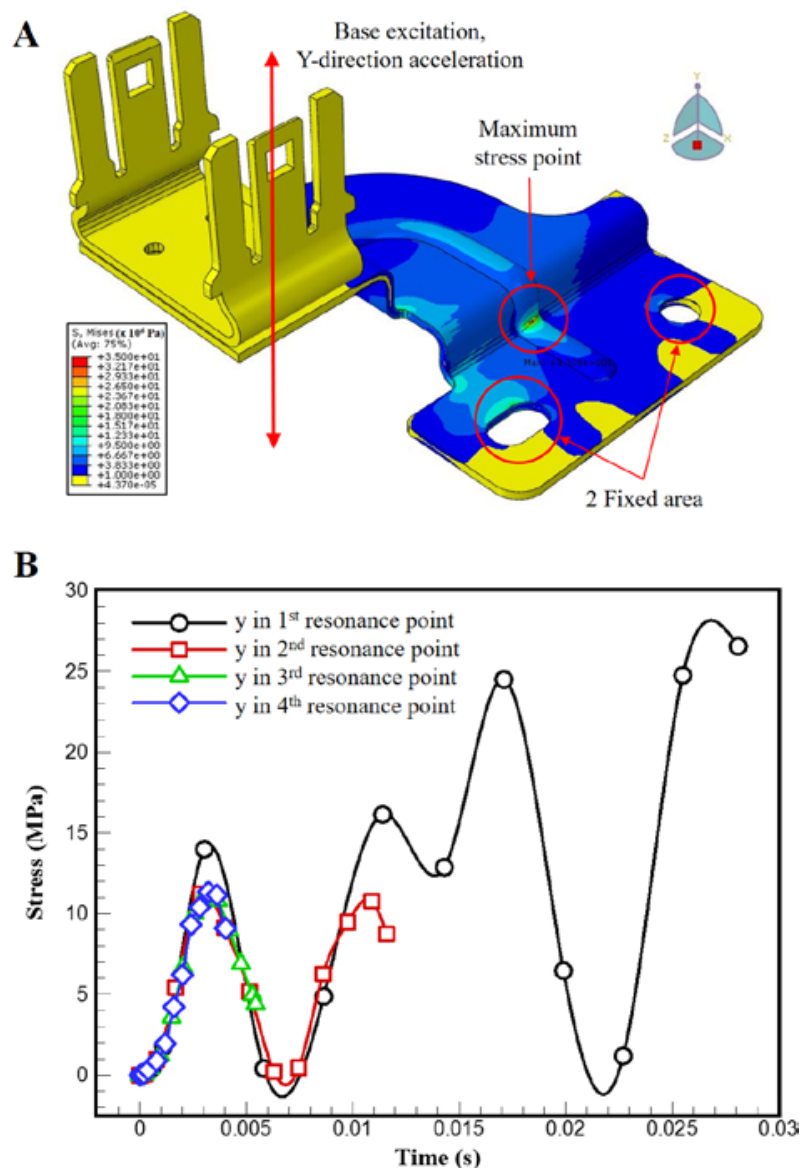

Figure 2. (A) Stress distribution on SL bracket surface and (B) stress variation on the maximum stress point of SL bracket

of the weight of brackets and additional process on structure legs, DL-M3 and DL-M4 model has $1.6 \mathrm{~mm}$ thickness (reduced $20 \%$ of base model bracket leg thickness) and 3mm filet radius with side flange bead and center bead as reinforce structures respectively.

\section{Results and discussion}

\subsection{Stress distribution on DL bracket}

Fig. 4 shows the resonant frequencies in modified brackets. The first point of resonance frequencies in DL-M1 to M4 are close to $180 \mathrm{~Hz}$. As the mode shapes number increases, the differences of resonance frequency are also increased. In $3^{\text {rd }}$ resonance point, DL-M1 and DL-M2 shows same resonance frequency over $1 \mathrm{kHz}$, by contrast, DL-M3 and DL-M4 shows decreased resonance frequency under
A

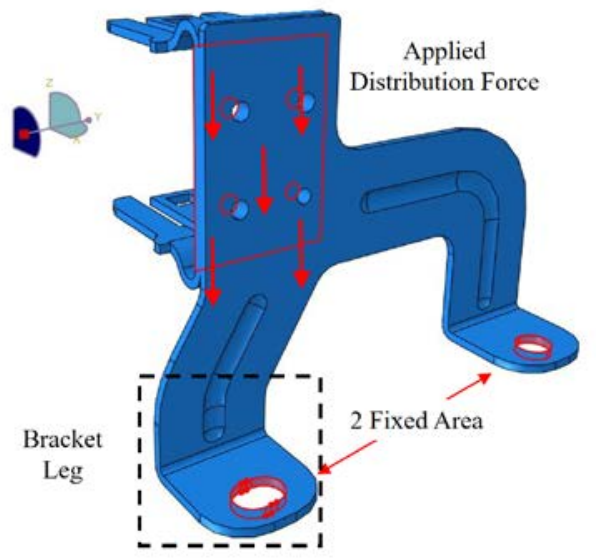

B

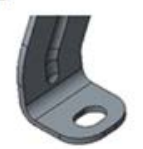

DL-M1

$\mathbf{R}=\mathbf{3}$

$\mathrm{T}=\mathbf{2 . 0}$

Original
C

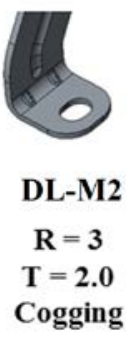

D
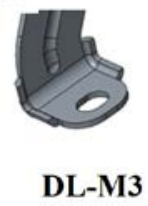

$\mathbf{R}=\mathbf{3}$

$\mathrm{T}=\mathbf{1 . 6}$

Flange Bead
E

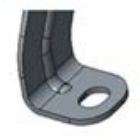

DL-M4

$\mathbf{R}=\mathbf{3}$

$\mathrm{T}=\mathbf{1 . 6}$
Figure 3. Schematics of (A) the DL bracket with (B-E) modified design on the bracket legs

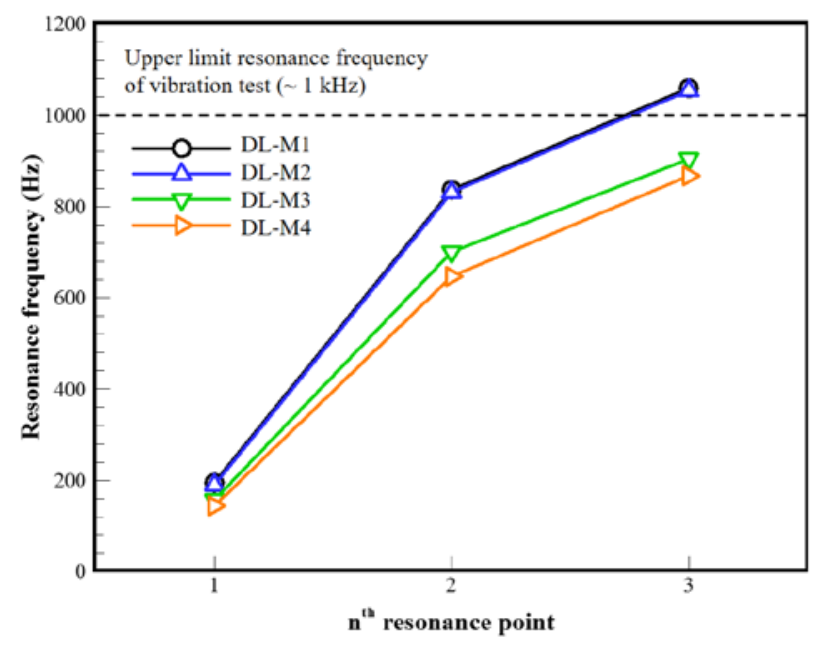

Figure 4. Resonance frequency of double legs bracket DLM0 to DL-M4 model around the $1,000 \mathrm{~Hz}$

$1 \mathrm{kHz}$ that is caused by decreased thickness of bracket legs. Table 2 shows the value of maximum stress in each directions ( $\mathrm{x}, \mathrm{y}$ and $\mathrm{z}$ ) and the mode numbers 1 to 3 . Depends on modified shape of bracket legs, the maximum stress on stress distribution are shown in fig.5B to 5E. The results of stress distribution show similar tendency that maximum stress is 
Table 2. The maximum stress of DL bracket with leg modified model DL-M1 to DL-M4 in each direction acceleration

\begin{tabular}{|c|c|c|c|}
\hline \multicolumn{4}{|c|}{ Maximum stress by vibration with specific direction (MPa) } \\
\hline Model & $\mathrm{x}$ & $\mathrm{Y}$ & $\mathrm{z}$ \\
\hline DL-M1 & $\begin{array}{c}17.04 \\
\text { (mode 1) }\end{array}$ & $\begin{array}{c}1.71 \\
\text { (mode 2) }\end{array}$ & $\begin{array}{c}3.74 \\
\text { (mode 1) }\end{array}$ \\
\hline DL-M2 & $\begin{array}{c}19.83 \\
\text { (mode 1) }\end{array}$ & $\begin{array}{c}1.57 \\
\text { (mode 2) }\end{array}$ & $\begin{array}{c}4.33 \\
\text { (mode 1) }\end{array}$ \\
\hline DL-M3 & $\begin{array}{c}40.84 \\
\text { (mode 1) }\end{array}$ & $\begin{array}{c}2.602 \\
\text { (mode 3) }\end{array}$ & $\begin{array}{c}9.75 \\
\text { (mode 1) }\end{array}$ \\
\hline DL-M4 & $\begin{array}{c}20.83 \\
\text { (mode 1) }\end{array}$ & $\begin{array}{c}2.88 \\
\text { (mode 3) }\end{array}$ & $\begin{array}{c}5.05 \\
\text { (mode 1) }\end{array}$ \\
\hline
\end{tabular}

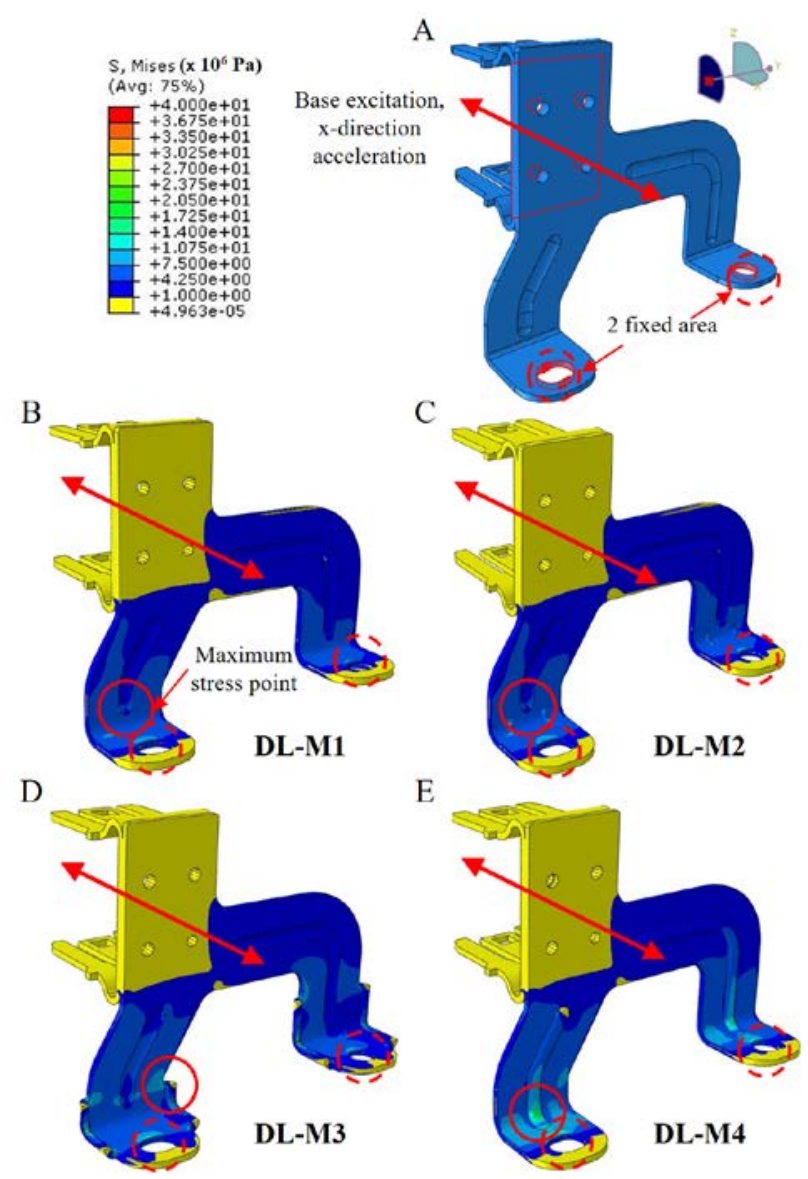

Figure 5. Maximum stress on DL bracket in each resonance frequency vibration condition.

observed in all bracket shapes in the x-direction of acceleration excitation in mode 1 . The leg thickness of DLM3 and DL-M4 brackets decrease by $20 \%$ compared to DLM1 bracket that shows low maximum stress less than $20 \mathrm{MPa}$ at hinge point. In the cases of DL-M2 with cogging process and DL-M4 with bead process brackets, there is no difference from DL-M1, however the local stress concentration is slightly increased near the cogging and bead region respectively. However, the maximum stress of DLM3 bracket is increased by 2.4 times rather than DL-M1 bracket, which causes stress concentration due to the interference with the bracket leg and the flange bead structure.

\section{Conclusions}

In this study, FEM vibration analysis is performed to bracket model with additional structures on bracket leg for enhancing the vibration durability. Before the FEM analysis, impact hammer and grid test is performed on single leg bracket to ensure the validity of the FEM model and to improve the accuracy of the analysis. The safety factor is considered in cyclic vibration conditions. The stress distribution result of single leg bracket shows necessity of relaxation of local stress concentration. As a result, DL-M4 bracket with $1.6 \mathrm{~mm}$ thickness and bead structure on legs is suitable to apply that shows $10.4 \%$ ratio of limited maximum stress/ yield stress below fatigue failure with saving the production cost.

\section{Acknowledgment}

This research is supported by "Reliability Technology Diffusion" through the Ministry of Trade, Industry and Energy (MOTIE) (N0001906, 2016) and "Basic Science Research Program" through the National Research Foundation of Korea (NRF) funded by the Ministry of Science, ICT \& Future Planning (2016R1C1B2012136).

\section{References}

(1) Tarak Gandhi, and Mohan Manubhai Trivedi : "Pedestrian Protection Systems: Issues, Survey, and Challenges", IEEE, Transactions on Intelligent Transportation Systems, Vol. 8, No. 3, pp 413-430, 2007

(2) Joel C. McCall, and Mohan M. Trivedi : "Driver Behavior and Situation Aware Brake Assistance for Intelligent Vehicles", Vol. 95, No. 2, pp 374-387, 2007

(3) T.J. Gordon, and M. Lidberg : "Automated driving and autonomous functions on road vehicles", Vol. 53, No. 7, 
pp 958-994, 2015

(4) Jianqiang Wang, Jian Wu, Xunjia Zheng, Daiheng Ni, and Keqiang $\mathrm{Li}$ : "Driving safety field theory modeling and its application in pre-collision warning system", Transportation Research Part C, Vol. 72, pp 306-324, 2016

(5) Albert G. Boulanger, Andrew C. Chu, Suzanne Maxx, and David L. Waltz : "Vehicle Electrification: Status and Issues", Proceedings of the IEEE, Vol. 99, No. 6, pp.1116-1138, 2011

(6) Youfan Hu, and Zhong Lin Wang : "Recent progress in piezoelectric nanogenerators as a sustainable power source in self-powered systems and active sensors", Nano Energy, Vol. 14, pp. 3-14, 2015

(7) Eduard Egusquiza, Carme Valero, Xingxing Huang, Esteve Jou, Alfredo Guardo, and Cristian Rodriguez : "Failure investigation of a large pump-turbine runner", Engineering Failure Analysis, Vol. 23, pp 27-34, 2012

(8) X. He, and S. O. Oyadiji : "Influence of adhesive characteristics on the transverse free vibration of single lap-jointed cantilevered beams", Vol. 119, pp 366-373, 2011

(9) S. L. Crouch, and A. M. Starfield, : Boundary element methods in solid mechanics, George Allen \& Unwin, London, 1983 